Apuntes Universitarios, 2020: 10(4), octubre-diciembre

ISSN: 2304-0335 DOI:https://doi.org/10.17162/au.v10i4.517

\title{
Procrastinación académica y ansiedad en estudiantes universitarios de Madre de Dios, Perú
}

\section{Academic procrastination and anxiety in university students of Madre de Dios}

\author{
Edwin Gustavo Estrada Araoz ${ }^{1}$; Helen Juddy Mamani Uchasara ${ }^{2}$ \\ Universidad Nacional Amazónica de Madre de Dios ${ }^{12}$ \\ ORCID ID: https://orcid.org/0000-0003-4159-934X ${ }^{1}$ \\ ORCID ID: $\underline{\text { https://orcid.org/0000-0003-1735-8570² }}$
}

Recibido: 14 de marzo de 2020

Aceptado: 15 de agosto de 2020

\begin{abstract}
Resumen
La presente investigación tuvo como objetivo determinar la relación que existe entre la procrastinación académica y la ansiedad en los estudiantes de la carrera profesional de Educación de la Universidad Nacional Amazónica de Madre de Dios. El enfoque de investigación fue cuantitativo, el diseño no experimental y el tipo correlacional. La muestra fue conformada por 220 estudiantes a quienes se les aplicó la Escala de Procrastinación Académica y la Escala de Autoevaluación de Ansiedad de Zung, instrumentos con la validez y confiabilidad requerida. Los resultados indican que el $48,2 \%$ de los estudiantes presentan un alto nivel de procrastinación académica, el 39\% tienen un nivel moderado de ansiedad y se determinó estadísticamente que existe una relación directa y significativa entre la procrastinación académica y la ansiedad ( $\mathrm{rs}=$ $0,359 ; \mathrm{p}=0,000<0,05)$. Se concluyó que cuanto más procrastinen los estudiantes, sus niveles de ansiedad serán más altos.
\end{abstract}

Palabras clave: Procrastinación académica; ansiedad; autorregulación; postergación; educación superior.

\footnotetext{
Abstract

${ }^{1}$ Correspondencia al autor

E-mail: edwin5721@outlook.com
}

The objective of this research was to determine the relationship that exists between academic procrastination and anxiety in the students of the Professional Career of Education of the National Amazonian University of Madre de Dios. The research focus was quantitative, non-experimental design and correlational type. The sample was made up of 220 students to whom the Academic Procrastination Scale and the Zung Anxiety Self-Assessment Scale were applied, instruments with 
the required validity and reliability. The results indicate that $48.2 \%$ of the students present a high level of academic procrastination, 39\% have a moderate level of anxiety and it was statistically determined that there is a direct and significant relationship between academic procrastination and anxiety ( $r s=0.359 ; p=0.000<0.05$ ). It was concluded that the more students procrastinate, their anxiety levels will be higher.

Keywords: Academic procrastination; anxiety; self-regulation; postponement; higher education.

\section{Introducción}

La vida universitaria genera un gran impacto en la actuar de los estudiantes a través de los diversos cambios y las adaptaciones que se dan (Domínguez, Villegas y Centeno, 2014; Domínguez, 2017). Muchos de los estudiantes pasan por momentos que requieren su máximo esfuerzo para desarrollar aprendizajes, obtener calificativos que les permitan continuar su carrera y culminarla satisfactoriamente para desenvolverse en el ámbito laboral (Mejía et al., 2018). Por ello, es necesario que los estudiantes desarrollen un repertorio de hábitos que les permitan gestionar y organizar su tiempo, realizar sus tareas de manera ordenada, repasar los temas desarrollados, prepararse para las exposiciones y exámenes y realizar oportunamente los trabajos encomendados por los docentes para obtener resultados de calidad (Garzón y Gil, 2017).

No obstante, es común observar que las mencionadas tareas y trabajos encargados en varias oportunidades pueden ser aplazados, lo que obstaculiza que se cumplan inmediatamente y genera problemas de índole personal y académico (Ramos et al., 2018; Domínguez, Villegas y Centeno, 2014). Este tipo de acciones orientadas a evadir, comprometerse a realizarlas más tarde o justificar la dilación y evitar la culpa hace referencia a la procrastinación (López et al., 2020; Quant y Sánchez, 2012; Hussain y Sultan, 2010), la cual es un comportamiento tan antiguo como la humanidad (García y Silva, 2019) y etimológicamente proviene del verbo latino procrastinare que significa “dejar algo para el día siguiente” (Rodríguez y Clariana, 2017).

En el ámbito educativo, la procrastinación académica es definida como la acción de dilatar de manera voluntaria e innecesaria el desarrollo de las tareas, por variadas causas (complejidad percibida, desagrado, etc.), que origina estrés y ansiedad (Domínguez, 2016). Es una conducta bastante común en los estudiantes universitarios puesto que entre el $80 \%$ y el $95 \%$ de los de ellos adopta este tipo de conductas en algún momento, el 75\% se considera a sí mismo procrastinador y el 50\% retrasa constantemente la dedicación a los estudios (Steel, 2007). 
Al respecto, un comportamiento bastante percibido en los estudiantes universitarios que procrastinan es que comienzan a estudiar mucho después de lo adecuado. Es posible que este atraso se origine por la discordancia de sus hábitos de estudio y sus propósitos, pero además porque la intención que tiene de estudiar está retrasada. "En consecuencia, estos se distraen fácilmente con otras actividades que no son las escolares, como por ejemplo las actividades sociales" (Álvarez, 2010, p.163).

Sin embargo, es necesario precisar que la postergación de actividades académicas no necesariamente provoca el incumplimiento de los trabajos o el logro de objetivos académicos, sino que en muchas ocasiones existe la tendencia a conseguirse bajo condiciones de elevado estrés y ansiedad (Alegre y Benavente, 2020). Bajo esa mirada, Pardo, Perilla y Salinas (2014) señalan que la dilación de actividades generará en el campo emocional tensión producida por los sentimientos de culpa y emociones asociadas a la presencia de altos niveles de ansiedad que ella genera. Lo expuesto sería corroborado por Alegre (2013) quien afirma que "la procrastinación no es solo un problema de la gestión o administración del tiempo, sino también es un proceso que implica una compleja interacción entre componentes cognitivos, afectivo-motivacionales y conductuales" (p.59).

Teniendo en cuenta lo manifestado, una investigación clasificó a la procrastinación académica en dos tipos, teniendo en cuenta su frecuencia y magnitud: la procrastinación esporádica y crónica (Schouwenburg, 2004). En ese sentido, la procrastinación esporádica es conocida como comportamiento dilatorio y se refiere a una forma de actuar puntual y se asocia con la postergación de ciertas actividades escolares debido más que todo a la pésima gestión del tiempo para organizar sus actividades, mientras que la procrastinación crónica es una costumbre generalizada en demorar o posponer la dedicación al estudio (Clariana, 2009).

Con relación a la ansiedad, es un trastorno común en los estudiantes universitarios debido a actividades propias de la educación universitaria tales como las participaciones y exposiciones en clases, la sobrecarga de trabajo y el poco tiempo para su ejecución, las exigencias de los docentes, la competencia entre pares así como la presión que tienen al rendir sus diferentes evaluaciones (Flores, Chávez y Aragón, 2016).

Cuando la ansiedad se presenta de manera esporádica puede considerarse como una respuesta de adaptación ante una amenaza o situaciones de estrés, ya que posibilita que las personas mejoren su desempeño, sin embargo, hay circunstancias en que dicha respuesta no resulta 
pertinente debido a que el estrés puede ser excesivo y superar los recursos con los cuales la persona dispone (Castillo, Chacón y Díaz, 2016), entonces, dicha ansiedad podría ser considerada patológica.

Bajo esa premisa, Spielberger (1972) menciona que la ansiedad patológica puede considerarse como rasgo y estado debido a que dicho trastorno evidencia síntomas perturbadores e irracionales en la persona. La ansiedad como rasgo es una cualidad de personalidad parcialmente constante que se muestra durante mucho tiempo y en variadas circunstancias, no obstante, la ansiedad como estado se refiere a un estado emocional pasajero y fluctuante en el tiempo (Reyes et al., 2017).

Todas las personas suelen experimentar ansiedad en algún momento de su vida, la cual es caracterizada por sentir principalmente "aprensión difusa, desagradable y vaga, que se manifiesta por la incapacidad de estar sentado o de pie durante un tiempo, y a menudo se acompaña de síntomas vegetativos como cefalea, diaforesis, taquicardia, opresión torácica, malestar epigástrico e inquietud" (Cardona et al., 2015, p.80).

Como se observa, los trastornos de ansiedad influyen negativamente en la calidad de vida de los estudiantes y en su desenvolvimiento académico, familiar, laboral y social, asimismo, promueven la aparición de otros trastornos psiquiátricos como el abuso de sustancias o la depresión y se relacionan con algunas enfermedades como la presión arterial alta, el asma, las úlceras gastrointestinales y el síndrome del intestino irritable (Lozano y Vega, 2013). En el ámbito académico, de no tratarse, podría ocasionar la desaprobación de cursos, disminución de sus niveles de bienestar, problemas emocionales o el retraso en el egreso y hasta el fracaso académico (Domínguez et al., 2017).

Actualmente, existen diversas investigaciones que reportaron que la procrastinación académica se relaciona directamente con la ansiedad, es decir, si los estudiantes que posponen las actividades y tareas que tienen que realizar oportunamente tienden a incrementar sus niveles de ansiedad, debido principalmente al poco tiempo que tienen para realizarlas y a su acumulación. (Saplavska y Jerkunkova, 2018; Pardo, Perilla y Salinas, 2014; Gil y Botello, 2018; Awuni, 2010; Kaur, 2018; Hussain \& Sultan, 2010).

Lo expuesto tiene sentido ya que varias posturas teóricas asumieron que las personas procrastinadoras tienen mayor posibilidad de experimentar ansiedad (Rothblum, Solomon y Murakami, 1986; Solomon y Rothblum, 1984; Ferrari, 1991), es decir, una serie de síntomas 
adaptativos pero que ponen en peligro su salud física y mental y no les permiten desenvolverse adecuadamente.

Asimismo, Gil, De Besa y Garzón (2020) y Domínguez (2016) afirmaron que cuando los estudiantes postergan la realización de sus trabajos suelen presentar días previos al tiempo máximo de entrega sensaciones de incomodidad y desagrado (estrés, ansiedad, preocupación y miedo). Estas reacciones psicológicas desfavorables para los estudiantes origina que los estudiantes tiendan a realizar sus actividades de manera apresurada y como es lógico, de baja calidad con la finalidad de tener una nota aprobatoria, aunque sea mínima.

En virtud a lo expuesto, surge la necesidad de conocer si en el contexto de la Universidad Nacional Amazónica de Madre de Dios (Perú), las conductas procrastinadoras se relacionan con los síntomas de ansiedad, asimismo, es menester identificar en qué medida los estudiantes de la carrera profesional de Educación procrastinan en el contexto académico y cuán ansiosos se encuentran. Del mismo modo, a nivel regional aún no hay investigaciones al respecto, por lo cual el estudio resulta pertinente y relevante para conocer la realidad por la que atraviesan nuestros estudiantes.

En vista de los resultados hallados, el área de psicopedagogía podrá plantear alternativas preventivas y, de ser el caso, correctivas, a través de planes de mejora, charlas, talleres, etc., con la finalidad de que los estudiantes en general vayan desterrando actitudes dilatorias innecesarias que les impidan mejorar su desempeño y calificaciones e influyan negativamente en su bienestar emocional. Por lo tanto, el objetivo de la presente investigación fue determinar la relación que existe entre la procrastinación académica y la ansiedad en los estudiantes de la carrera profesional de Educación de la Universidad Nacional Amazónica de Madre de Dios.

\section{Metodología}

El enfoque de esta investigación fue cuantitativo ya que se realizó la recolección de datos y el análisis de datos para responder a preguntas de investigación y probar las hipótesis definidas previamente, haciendo uso de la estadística para determinar el comportamiento de la muestra (Sánchez, Reyes y Mejía, 2018); el diseño fue no experimental, ya que ambas variables no fueron manipuladas, sino fueron observadas tal como se dieron en su entorno y luego se analizaron (Hernández, Fernández y Baptista, 2014) y el tipo fue correlacional debido a que se buscó descubrir 
las relaciones existentes entre las variables funcionamiento familiar y adicción a redes sociales (Bisquerra, 2009).

La población de estudio estuvo conformada por 515 estudiantes pertenecientes a la carrera profesional de Educación, en sus tres especialidades: Inicial y Especial, Primaria e Informática y Matemática y Computación, matriculados en el ciclo 2020-I y la muestra quedó constituida por 220 estudiantes. En la tabla 1 se detalla la distribución de la población y la muestra.

\section{Tabla 1}

Distribución de la población y la muestra

\begin{tabular}{lcc}
\hline \multicolumn{1}{c}{ Especialidades } & Población & Muestra \\
\hline Inicial y Especial & 253 & 109 \\
Primaria e Informática & 179 & 76 \\
Matemática y Computación & 83 & 35 \\
Total & 515 & 220 \\
\hline
\end{tabular}

Fuente: Dirección Universitaria de Asuntos Académicos

La técnica utilizada fue la encuesta y los instrumentos para recoger los datos fueron la Escala de Procrastinación Académica y la Escala de Autoevaluación de Ansiedad de Zung. La Escala de Procrastinación Académica fue diseñada por Busko (1998) y adaptada en el Perú por Álvarez (2010), evalúa la tendencia de los estudiantes a la procrastinación académica, está conformada por 16 ítems de tipo Likert (nunca, pocas veces, a veces, casi siempre y siempre) y evalúa 2 dimensiones: autorregulación académica (ítems del 1 al 11) y postergación de actividades (ítems del 12 al 16). Sus propiedades métricas fueron determinadas también mediante el proceso de validación y confiabilidad. La validación de contenido fue realizada a través de la técnica de juicio de expertos, obteniéndose luego de su evaluación un coeficiente V de Aiken de 0,916; lo que indica que la escala tiene muy buena validez. En cuanto a la confiabilidad, fue obtenida a través una prueba piloto realizada a 20 estudiantes, obteniéndose un coeficiente Alfa de Cronbach de 0,881; lo que significa que el instrumento es altamente confiable.

Con relación a la Escala de Autoevaluación de Ansiedad de Zung, fue diseñada por Zung (1971), está conformada por 20 ítems de tipo Likert (nunca, a veces, casi siempre y siempre) y mide 2 dimensiones: síntomas somáticos (ítems del 1 al 15) y síntomas cognoscitivos- afectivos (ítems del 16 al 20). Sus propiedades psicométricas también fueron determinadas mediante los 
procesos de validación y confiabilidad. El coeficiente $\mathrm{V}$ de Aiken obtenido mediante la validación de expertos fue de 0,842 y el coeficiente Alfa de Cronbach obtenido, tras la realización de una prueba piloto, fue de 0,834 ; lo cual quiere decir que la escala presenta buena confiabilidad y validez.

Con relación a la recolección de datos, se dio entro los meses de junio y julio del año 2020. Para ello se contactó a través del Whatsapp a los estudiantes con la finalidad de comunicarles el propósito de la investigación y enviarles el enlace para que puedan acceder al formulario de Google. Una vez que ellos accedieron al mencionado formulario, leyeron las orientaciones, brindaron su consentimiento para participar de manera voluntaria en la investigación (marcando con un check) y luego procedieron a desarrollar los cuestionarios. El acceso a la encuesta fue cerrado tras recibir las 220 respuestas y después fueron consolidadas en una base de datos donde se realizó el proceso de calificación de acuerdo a las normas de corrección.

Para realizar el análisis estadístico se recurrió al Software SPSS versión 22, donde los resultados fueron sistematizados a través de tablas de frecuencia. Para el análisis inferencial se utilizó la prueba no paramétrica rho de Spearman debido al nivel de medición de las variables (ordinal), su naturaleza (categórica) y a que no se ajustaron a la distribución normal. Finalmente, para la significancia estadística, se consideró un valor menor o igual a 0,05.

\section{Análisis de datos y discusión}

Los resultados que se exponen a continuación son el resultado del tratamiento estadístico realizado a los datos obtenidos a mediante la recolección de datos. Los resultados expuestos en la tabla 2 revelan en cuanto a la procrastinación, que el $48 \%$ de los estudiantes presentaron niveles altos, el 23,2\% tuvo niveles moderados, el 12,3\% evidenciaron muy altos niveles, el 10,5\% tenían niveles bajos y el 5,9\% mostraron muy bajos niveles. Respecto a la ansiedad, el 39,1\% presentaron niveles moderados, el 35\% tenía bajos niveles, el 13,2\% evidenciaron altos niveles, el 9,1\% tenían muy altos niveles y el 3,6\% presentaron muy bajos niveles.

\section{Tabla 2}

Resultados descriptivos de las variables procrastinación académica y ansiedad

\begin{tabular}{lcccc}
\multirow{2}{*}{ Categorías } & \multicolumn{2}{c}{ Procrastinación académica } & \multicolumn{2}{c}{ Ansiedad } \\
\cline { 2 - 5 } & $\mathrm{N}$ & $\%$ & $\mathrm{n}$ & $\%$ \\
\hline Muy bajo & 13 & 5,9 & 8 & 3,6
\end{tabular}




\begin{tabular}{lcccc} 
Bajo & 23 & 10,5 & 77 & 35,0 \\
Moderado & 51 & 23,2 & 86 & 39,1 \\
Alto & 106 & 48,2 & 29 & 13,2 \\
Muy alto & 27 & 12,3 & 20 & 9,1 \\
Total & 220 & 100,0 & 220 & 100,0 \\
\hline
\end{tabular}

Fuente: Base de datos

Los datos de la tabla 3 evidencian la prueba de normalidad Kolmogorov - Smirnov, donde podemos observar que las puntuaciones no se ajustaron a la distribución normal $(\mathrm{p}=0,000<0,05)$. Por lo tanto, viendo la naturaleza de las variables (categóricas), su nivel de medición (ordinal) así como la bondad de ajuste, se decidió utilizar la prueba no paramétrica rho de Spearman.

\section{Tabla 3}

Prueba de normalidad Kolmogorov - Smirnov

\begin{tabular}{lccc}
\multirow{2}{*}{ Variables y dimensiones } & \multicolumn{3}{c}{ Kolmogorov-Smirnov $^{\mathrm{a}}$} \\
\cline { 2 - 4 } & Estadístico & $\mathrm{gl}$ & Sig. \\
\hline Procrastinación académica &, 140 & 220 &, 000 \\
Ansiedad &, 102 & 220 &, 000 \\
\hline
\end{tabular}

Fuente: Base de datos

Según los datos expuestos en la tabla 4, el coeficiente de correlación rho de Spearman entre las variables procrastinación académica y ansiedad fue de 0,359 con un p-valor inferior al nivel de significancia $(\mathrm{p}=0,000<0,05)$, por ello, se rechazó la hipótesis nula y se aceptó la hipótesis alterna, la cual indicaba la existencia de una relación directa y significativa entre ambas variables. Por lo expuesto, se determinó que mientras los estudiantes procrastinen con mayor frecuencia, sus niveles de ansiedad serán mayores. 
Tabla 4

Correlación entre la procrastinación académica y la ansiedad

\begin{tabular}{|c|c|c|c|c|}
\hline & & & $\begin{array}{c}\text { Procrastinación } \\
\text { académica }\end{array}$ & Ansiedad \\
\hline \multirow{6}{*}{$\begin{array}{c}\text { Rho de } \\
\text { Spearman }\end{array}$} & \multirow{3}{*}{$\begin{array}{l}\text { Procrastinación } \\
\text { académica }\end{array}$} & Coeficiente de correlación & 1,000 &, $359^{* *}$ \\
\hline & & Sig. (bilateral) & . &, 000 \\
\hline & & $\mathrm{N}$ & 220 & 220 \\
\hline & \multirow{3}{*}{ Ansiedad } & Coeficiente de correlación &, $359^{* *}$ & 1,000 \\
\hline & & Sig. (bilateral) &, 000 & . \\
\hline & & $\mathrm{N}$ & 220 & 220 \\
\hline
\end{tabular}

**. La correlación es significativa en el nivel 0,01 ( 2 colas).

De acuerdo a los datos expuestos en la tabla 5, el coeficiente de correlación rho de Spearman entre la dimensión autorregulación académica y la variable ansiedad fue de -0,412 con un p-valor inferior al nivel de significancia $(\mathrm{p}=0,000<0,05)$, por lo tanto, se rechazó la hipótesis nula y se aceptó la hipótesis alterna, la cual indicaba la existencia de una relación inversa y significativa entre la dimensión y variable analizada. Por lo tanto, se determinó que mientras los estudiantes autorregulen y organicen sus actividades académicas, sus niveles de ansiedad serán menores.

Tabla 5

Correlación entre la autorregulación académica y la ansiedad

\begin{tabular}{cclcc} 
& & & $\begin{array}{c}\text { Autorregulación } \\
\text { académica }\end{array}$ & Ansiedad \\
\hline \multirow{3}{*}{ Rho de } & Autorregulación & Coeficiente de correlación & 1,000 &,$- 412^{* *}$ \\
\cline { 3 - 5 } Spearman & académica & Sig. (bilateral) & $\cdot$ &, 000 \\
& & $\mathrm{~N}$ & 220 & 220 \\
\cline { 2 - 5 } & \multirow{2}{*}{ Ansiedad } & Coeficiente de correlación &,$- 412^{* *}$ & 1,000 \\
& & Sig. (bilateral) &, 000 & $\cdot$ \\
& & $\mathrm{N}$ & 220 & 220 \\
\hline
\end{tabular}

**. La correlación es significativa en el nivel 0,01 (2 colas)

Los hallazgos expuestos en la tabla 6 indican que el coeficiente de correlación rho de Spearman entre la dimensión postergación de actividades y la variable ansiedad fue de 0,338 con un p-valor inferior al nivel de significancia $(\mathrm{p}=0,003<0,05)$, por lo tanto, se rechazó la hipótesis nula y se aceptó la hipótesis alterna, la cual indicaba la existencia de una relación directa y significativa entre la dimensión y variable analizada. Por lo tanto, se estableció que cuanto más posterguen los estudiantes sus actividades académicas, sus niveles de ansiedad serán mayores. 


\section{Tabla 6}

Correlación entre la postergación de actividades y la ansiedad

\begin{tabular}{cclcc} 
& & & $\begin{array}{c}\text { Postergación de } \\
\text { actividades }\end{array}$ & Ansiedad \\
\hline \multirow{3}{*}{ Rho de } & Postergación de & Coeficiente de correlación & 1,000 &, $338^{* *}$ \\
Spearman & Sig. (bilateral) & $\cdot$ &, 003 \\
\cline { 2 - 5 } & \multirow{2}{*}{ Ansiedad } & Coeficiente de correlación & 220 & 220 \\
& & Sig. (bilateral) &, $338^{* *}$ & 1,000 \\
& & $\mathrm{~N}$ & 220 & $\cdot$ \\
& & & 220 & 203 \\
\hline
\end{tabular}

**. La correlación es significativa en el nivel 0,01 (2 colas).

\section{Discusión}

El objetivo de la presente investigación fue determinar la relación que existe entre la procrastinación académica y la ansiedad en los estudiantes de la carrera profesional de Educación de la Universidad Nacional Amazónica de Madre de Dios Perú. Un primer hallazgo indica que los estudiantes se caracterizan por presentar un alto nivel de procrastinación académica, es decir, suelen aplazar de manera voluntaria e innecesaria las actividades académicas encomendadas por el docente (tareas, foros, lecturas, exámenes, etc.), dejándolas para el último momento, lo cual pone en riesgo sus calificaciones y la continuidad de sus estudios. Este hallazgo es corroborado por diferentes investigaciones quienes reportaron que los estudiantes postergaban sus actividades para dedicarse a actividades menos trascendentes (Córdova y Alarcón, 2019; Gil y Botello, 2018; Domínguez, 2017).

Al respecto, es necesario mencionar que la procrastinación académica "es el resultado de carencias en la autorregulación, que conduce al individuo a la dilatación voluntariamente pero inconsciente sobre el inicio y consumación de las tareas o actividades previstas, a pesar de conocer las consecuencias negativas de postergar" (Atalaya y García, 2019, p.368). Por ello, es necesario desarrollar en los estudiantes estrategias autorreguladoras para que gestionen su tiempo y efectúen sus trabajos oportunamente.

En cuanto a la ansiedad, se identificó que los estudiantes presentan niveles moderados, es decir, algunos de ellos presentan síntomas adaptativos como cefalea, opresión torácica, malestar epigástrico e inquietud frente a los estresores propios de las actividades académicas. Este hallazgo 
coincide con los reportes Cardona, et al. (2015) y Britos (2017) quienes concluyeron que los estudiantes universitarios evaluados presentaban niveles medios de ansiedad traducidos en señales de alerta que advierten peligros y les permite tomar medidas para afrontarlas.

Con relación a lo expuesto se puede precisar que la ansiedad tiende a manifestarse mediante respuestas poco agradables para los estudiantes, ya sea a nivel cognitivo, fisiológico y motor (Flores, Chávez y Aragón, 2016). Si los estudiantes no manejan adecuadamente estas sensaciones no podrán afrontar adecuadamente los problemas que se les presenten y afectará indudablemente en su desenvolvimiento en clases y otros contextos (familiares, personales, amicales o laborales). Por otro lado, un hallazgo revelador indica que la procrastinación académica está relacionada de manera directa y significativa con la ansiedad de los estudiantes, es decir, mientras los estudiantes posterguen con mayor frecuencia las actividades académicas de manera voluntaria, sus niveles de ansiedad se incrementarán, lo que a su vez afectará su bienestar personal y desde luego, su rendimiento académico.

Lo expuesto anteriormente, es corroborado por diversas investigaciones (Saplavska y Jerkunkova, 2018; Pardo, Perilla y Salinas, 2014; Gil y Botello, 2018; Awuni, 2010; Kaur, 2018; Hussain \& Sultan, 2010) que determinaron en sus investigaciones realizadas con estudiantes universitarios que la procrastinación académica se relaciona de manera directa con el fracaso, el estrés y la ansiedad. Por ello, la labor tutorial en las universidades será necesario para fomentar en los estudiantes la autorregulación, los hábitos de estudio y la organización del tiempo para mejorar su desenvolvimiento y evitar que incurran en la postergación de actividades. En este orden de ideas, Garzón y Gil (2017) afirman que los estudiantes con mejor rendimiento académico se caracterizan principalmente porque "tienen metas más altas, usan con mayor frecuencia estrategias de elaboración y organización de la información, manejo del tiempo y el ambiente de estudio, estrategias metacognitivas autorregulatorias y un mayor esfuerzo de autorregulación” (p.311).

Lo manifestado explica que la procrastinación académica estaría asociada esencialmente con los bajos niveles de autorregulación y la gestión del tiempo, por ello muchos estudiantes generalmente no fijan metas a corto y mediano plazo ni organizan sus actividades por lo que resulta desordenada la manera en que llevan a cabo sus actividades académicas. Como consecuencia de lo manifestado, tienen que lidiar finalmente con la acumulación de los diferentes trabajos que se les 
encarga que sumados al estrés y ansiedad que ello genera, tienden a realizarla de forma poco adecuada y en otros casos, a no cumplirlas.

De acuerdo a la literatura analizada, la procrastinación académica es una variable que debe seguir profundizándose pues, como se pudo ver, afecta el desenvolvimiento académico y personal de los estudiantes. Los hallazgos corroboran lo expuesto ya que determinó su relación de manera directa con la ansiedad que ellos manifestaban. Estos resultados pueden ser útiles para el diseño y ejecución de programas enfocados en disminuir su prevalencia.

Finalmente, es necesario precisar que la presente investigación tuvo algunas limitaciones como la cantidad de la muestra, la cual no permite la generalización de los hallazgos, restringiéndolos al presente grupo de estudiantes universitarios, por ello, sería importante aumentar la cantidad de estudiantes participantes de otras carreras profesionales para poder confirmar y generalizar los resultados. Asimismo, no se consideró los datos sociodemográficos para analizar los hallazgos por lo que sería importante que futuras investigaciones realicen comparaciones, principalmente en función al sexo, ciclo, grupo etario y carrera profesional para tener una mirada más precisa de la problemática abordada.

\section{Conclusión}

Los hallazgos del presente estudio permitieron demostrar la existencia de una relación directa y significativa entre la procrastinación académica y la ansiedad en los estudiantes de la carrera profesional de Educación de la Universidad Nacional Amazónica de Madre de Dios. Por tal motivo, cuanto más procrastinen los estudiantes y pospongan sus actividades académicas, los niveles de ansiedad que se generen serán mayores. Bajo esa premisa, es necesario que el área de psicopedagogía de la universidad diseñe programas que les permita a los estudiantes gestionar su tiempo de manera pertinente para que realicen sus actividades oportunamente, dejando de lado las prácticas dilatorias y así no estén ansiosos días previos a la entrega de sus trabajos y desarrollo de exámenes. 


\section{Referencias}

Alegre, A. (2013). Autoeficacia y procrastinación académica en estudiantes universitarios de Lima Metropolitana. Propósitos y $\quad$ Representaciones, $\quad$ 1(2), http://dx.doi.org/10.20511/pyr2013.v1n2.29

Alegre, A. y Benavente, D. (2020). Análisis Psicométrico de la Escala Adaptada de Procrastinación de Tuckman (APTS). Propósitos y Representaciones, 8(2), e562. http://dx.doi.org/10.20511/pyr2020.v8n2.562

Álvarez, O. (2010). Procrastinación general y académica en una muestra de estudiantes de secundaria de Lima metropolitana. Persona, 13, 159-177. Recuperado de https://revistas.ulima.edu.pe/index.php/Persona/article/viewFile/270/257

Atalaya, C. y García, L. (2019). Procrastinación: Revisión teórica. Revista de Investigación en Psicología, 22(2), 363-378. https://doi.org/10.15381/rinvp.v22i2.17435

Awuni, J. (2010). Correlates of course anxiety and academic procrastination in higher education. Global Journal of Educational Research, 10(1), 55-65. Recuperado de https://www.ajol.info/index.php/gjedr/article/view/79048

Bisquerra, R. (2009). Metodología de la Investigación Educativa. (2a ed.), Madrid, España: La Muralla S. A.

Britos, M. (2017). Uso de internet y ansiedad en adolescentes escolarizados de una institución educativa gubernamental, año 2016. Revista Científica de la UCSA, 4(3), 34-47. Recuperado de $\quad$ https://ucsa.edu.py/yeah/wp-content/uploads/2017/12/4.-AO_Britos-Esquivel-M_34$\underline{47 . p d f}$

Busko, D. (1998). Causes and consequences of perfectionism and procrastination: A structural equation model (Tesis de posgrado no publicada). University of Guelph, Ontario, Canadá.

Cardona, J., Pérez, D., Rivera, S. y Gómez, J. (2015). Prevalencia de ansiedad en estudiantes universitarios. Diversitas, 11(1), 79-89. https://doi.org/10.15332/s1794-9998.2015.0001.05

Castillo, C., Chacón, T. y Díaz, G. (2016). Ansiedad y fuentes de estrés académico en estudiantes de carreras de la salud. Investigación en Educación Médica, 5(20), 230-237. https://doi.org/10.1016/j.riem.2016.03.001

Clariana, M. (2009). Procrastinació acadèmica. Barcelona, España: Servei de publicacions de la Universitat Autònoma de Barcelona. 
Córdova, P. y Alarcón, A. (2019). Hábitos de estudio y procrastinación académica en estudiantes universitarios en Lima Norte. CASUS, Revista de Investigación y Casos en Salud, 4(1). https://doi.org/10.35626/casus.1.2019.161

Domínguez, S. (2016). Datos normativos de la Escala de Procrastinación Académica en estudiantes de psicología de Lima. Revista Evaluar, 16(1). https://doi.org/10.35670/16674545.v16.n1.15715

Domínguez, S. (2017). Prevalencia de procrastinación académica en estudiantes universitarios de lima metropolitana y su relación con variables demográficas. Revista de Psicología, 7(1), 8195. Recuperado de http://revistas.ucsp.edu.pe/index.php/psicologia/article/view/49

Domínguez, S., Calderón, G., Alarcón, D. y Navarro, J. (2017). Relación entre ansiedad ante exámenes y rendimiento en exámenes en universitarios: análisis preliminar de la diferencia según asignatura. Revista Digital de Investigación en Docencia Universitaria, 11(1), 163173. https://doi.org/10.19083/ridu.11.492

Domínguez, S., Villegas, G. y Centeno, S. (2014). Procrastinación académica: validación de una escala en una muestra de estudiantes de una universidad privada. Liberabit, 20(2), 293-304. Recuperado de https://www.redalyc.org/pdf/686/68632617010.pdf.

Ferrari, J. (1991). Self-handicapping by procrastinators: Protecting selfesteem, social-esteem, or both? Journal of Research in Personality, 25, 245-261. https://doi.org/10.1016/0092$\underline{6566(91) 90018-\mathrm{L}}$

Flores, M., Chávez, M. y Aragón, L. (2016). Situaciones que generan ansiedad en estudiantes de Odontología. Journal of Behavior, Health \& Social Issues, 8(2), 35-41. https://doi.org/10.1016/j.jbhsi.2016.11.004

García, V. y Silva, M. (2019). Procrastinación académica entre estudiantes de cursos en línea. $\begin{array}{llllll}\text { Validación de un } & \text { cuestionario. } & \text { Apertura 122-137. }\end{array}$ https://dx.doi.org/10.32870/ap.v11n2.1673

Garzón, A. y Gil, J. (2017). Gestión del tiempo y procrastinación en la educación superior. Universitas Psychologica, 16(3), 1-13. https://doi.org/10.11144/Javeriana.upsy16-3.gtpe

Gil, J., De Besa, M. y Garzón, A. (2020). ¿Por qué procrastina el alumnado universitario? Análisis de motivos y caracterización del alumnado con diferentes tipos de motivaciones. Revista de Investigación Educativa, 38(1), 183-200. https://doi.org/10.6018/rie.344781 
Gil, L. y Botello, V. (2018). Procrastinación académica y ansiedad en estudiantes de Ciencias de la Salud de una Universidad de Lima Norte. CASUS, Revista de Investigación y Casos en Salud, 3(2), 89-96. https://doi.org/10.35626/casus.2.2018.75

Hernández, R. Fernández, C. y Baptista, P. (2014) Metodología de la Investigación (6ta ed.), México D.F., México: Mc Graw-Hill.

Hussain, I. \& Sultan, S. (2010). Analysis of procrastination among university students. Procedia Social and Behavioral Sciences, 5, 1897-1904. https://doi.org/10.1016/j.sbspro.2010.07.385

Kaur, S. (2018). A study of procrastination in relation to anxiety among female college students in terms of locale and family structure. International Journal of Education and Management Studies, $\quad 8(1), \quad$ 71-75. $\quad$ Recuperado de $\quad$ http://www.ischolar.in/index.php/injems/article/view/173951

López, A., Toca, L., Gonzáles, J., Matías, B. y Alonso, M. (2020). Reducción de la procrastinación académica mediante la Terapia de Aceptación y Compromiso: un estudio piloto. Clínica Contemporánea, 11(e4), 1-16. https://doi.org/10.5093/cc2020a3

Lozano, A. y Vega, J. (2013). Evaluación psicométrica y desarrollo de una versión reducida de la nueva escala de ansiedad en una muestra hospitalaria de Lima, Perú. Revista Peruana de Medicina Experimental y Salud Publica, 30(2), 212-219. https://doi.org/10.17843/rpmesp.2013.302.193

Mejía, C., Ruiz, F., Benites, D. y Pereda, W. (2019). Factores académicos asociados a la procrastinación. Revista Cubana de Medicina General Integral, 34(3). Recuperado de http://www.revmgi.sld.cu/index.php/mgi/article/view/954

Pardo, D., Perilla, L. y Salinas, C. (2014). Relación entre procrastinación académica y ansiedadrasgo en estudiantes de psicología. Cuadernos Hispanoamericanos de Psicología, 14(1), 3144. Recuperado de https://revistas.unbosque.edu.co/index.php/CHP/article/view/1343/965.

Quant, D. y Sánchez, A. (2012). Procrastinación, procrastinación académica: concepto e implicaciones. Revista Vanguardia Psicológica, 3(1), 45-59.

Ramos, C., Jadán, J., Paredes, L., Bolaños, M. y Gómez, A. (2018). Procrastinación, adicción al internet y rendimiento académico de estudiantes universitarios ecuatorianos. Estudios Pedagógicos, 43(3), 275-289. https://doi.org/10.4067/S0718-07052017000300016 
Reyes, C., Monterrosas, A., Navarrete, A., Acosta, E. y Torruco, U. (2017). Ansiedad de los estudiantes de una facultad de medicina mexicana, antes de iniciar el internado. Investigación en Educación Médica, 6(21), 42-46. https://doi.org/10.1016/j.riem.2016.05.004

Rodríguez, A. y Clariana, M. (2017). Procrastinación en estudiantes universitarios: Su relación con la edad y el curso académico Revista Colombiana de Psicología, 26(1), 45-60. http://dx.doi.org/10.15446/rcp.v26n1.53572

Rothblum, E., Solomon, L. y Murakami, J. (1986). Affective, cognitive, and behavioral differences between high and low procrastinators. Journal of Counseling Psychology, 33(4), 387-394. https://doi.org/10.1037/0022-0167.33.4.387

Sánchez, H., Reyes, C. y Mejía, K. (2018). Manual de términos en investigación científica, tecnológica y humanística. Universidad Ricardo Palma: Lima. Recuperado de http://repositorio.urp.edu.pe/handle/URP/1480

Saplavska, J. \& Jerkunkova, A. (2018). Academic procrastination and anxiety among students. Engineering for rural development - International Scientific Conference 2018, 1192-1197. https://doi.org/10.22616/ERDev2018.17.N357

Schouwenburg, H (2004). Procrastination in Academic Settings: General Introduction. In H. C. Schouwenburg, C. H., Lay, T. A., Pychyl, \& J. R. Ferrari (eds.), Counseling the procrastinator in academic settings (p. 3-17). American Psychological Association. https://doi.org/10.1037/10808-001

Solomon, L. y Rothblum, E. (1984). Academic procrastination: Frequency and cognitivebehavioral correlates. Journal of Counseling Psychology, 31(4), 503-509. https://doi.org/10.1037/0022-0167.31.4.503

Spielberger, C. (1972). Anxiety as an emotional state. En C.D. Spielberger (Ed.), Anxiety Behavior (pp. 23-49). New York: Academic Press.

Steel, P. (2007). The nature of procrastination: A metanalytic and theoretical review of quintessential self-regulatory failure. Psychological Bulletin, 133(1), 65-94. https://doi.org/10.1037/0033-2909.133.1.65

Zung, W. (1971). A rating instrument for anxiety disorders. Psychosomatics, 12(6), 371-379. https://doi.org/10.1016/S0033-3182(71)71479-0 